\title{
Formation of quantum structures on a single nanotube by modulating hydrogen adsorption
}

\author{
O. Gülseren, ${ }^{1}$ T. Yildirim, ${ }^{2}$ and S. Ciraci ${ }^{1}$ \\ ${ }^{1}$ Department of Physics, Bilkent University, Ankara 06800, Turkey \\ ${ }^{2}$ NIST Center for Neutron Research, National Institute of Standards and Technology, Gaithersburg, Maryland 20899, USA
}

(Received 17 October 2002; revised manuscript received 2 June 2003; published 18 September 2003)

\begin{abstract}
Using first-principles density functional calculations we showed that quantum structures can be generated on a single carbon nanotube by modulating the adsorption of hydrogen atoms. The band gap of the hydrogen-free zone of the tube widens in the adjacent hydrogen covered zone. The sudden variation of the band gap leads to band offsets at the conduction- and valence-band edges. At the end, the band gap of the whole system is modulated along the axis of the tube, which generates quantum wells or quantum dots. Specific electronic states are confined in these quantum wells. The type and radius of the nanotube and the extent and sequence of hydrogen-free and hydrogen-covered zones can provide several options to design a desired optoelectronic nanodevice.
\end{abstract}

DOI: 10.1103/PhysRevB.68.115419

PACS number(s): 73.22.-f, 73.40.Lq, 61.48.+c, 73.20.Hb

\section{INTRODUCTION}

Novel properties and fundamental effects of electrons have been revealed in lower-dimensional quantum structures, such as multiple quantum wells, quantum dots, etc. During last two decades several new electronic devices have been developed from these quantum structures. ${ }^{1}$ Fabricating even smaller and more efficient devices is now the prime drive towards nanotechnology and molecular electronics. One of the grand challenges of research on carbon nanotubes ${ }^{2}$ has been the realization of nanometer optoelectronic devices. To this end single-wall carbon nanotubes (SWNT's) have been explored actively with the motivation of finding a new device application. ${ }^{3-7}$ In particular, important effects of the adsorption of foreign atoms (i.e., functionalization) on the electronic properties have been observed. Remarkable changes in the electrical resistance and thermoelectric power of a SWNT upon exposure to gaseous molecules such as $\mathrm{NO}_{2}$, $\mathrm{NH}_{3}$, and $\mathrm{O}_{2}$ have been exploited to form chemical sensors. ${ }^{3,4}$

In this paper we present a quantum well structure generated by the modulating adsorption of hydrogen atoms on a semiconducting SWNT. It is known that a SWNT can be either a metal or a semiconductor depending on its chiral vector and radius, but may become a semiconductor even with a larger band gap, when it is exohydrogenated. ${ }^{8,9}$ Hence, our idea of forming quantum structures on a SWNT exploits this important effect of hydrogenation. Electrons or holes can be confined in a hydrogen-free region of SWNT's sandwiched between two regions, which are uniformly hydrogenated.

\section{MODEL AND METHOD OF CALCULATION}

These findings are obtained from extensive state-of-theart, first-principle density functional calculations ${ }^{10}$ within the generalized gradient approximation ${ }^{11}$ for fully optimized atomic positions as well as the lattice constant. The predictive power of the density functional theory was proved in earlier studies related to the atomic structure, energetics, and electronic structure of various carbon nanotubes. ${ }^{8,9,12} \mathrm{We}$ have generated the superlattice structure on a single $(8,0)$ SWNT, on which a part (or a zone) of $l$ unit cells [specified by $\left(\mathrm{C}_{32}\right)_{l}$ ] is kept clean, while the adjacent part of $q$ unit cells [specified by $\left(\mathrm{C}_{32} \mathrm{H}_{32}\right)_{q}$ ] is fully hydrogenated. We also assume that the supercell $\left[\left(\mathrm{C}_{32}\right)_{l}\left(\mathrm{C}_{32} \mathrm{H}_{32}\right)_{q}\right]$ is repeated periodically to form a superlattice. Such superlattice structures generated on a $(8,0)$ tube are illustrated in Fig. 1. Calculations have been carried out within periodically repeating supercell geometry because of the necessity of using periodic boundary conditions with the plane-wave method. The tetragonal supercell has lattice constants $a_{s}, b_{s}$, and $c_{s}$, where $a_{s}$ and $b_{s}$ are chosen such that the interaction between nearest-neighbor tubes is negligible (the minimum C-C distance between two nearest-neighbor tubes is taken as $7 \AA$ ). The lattice constant $c_{s}$ along the tube axis is taken to be equal to the periodicity of the superlattice (or one period of

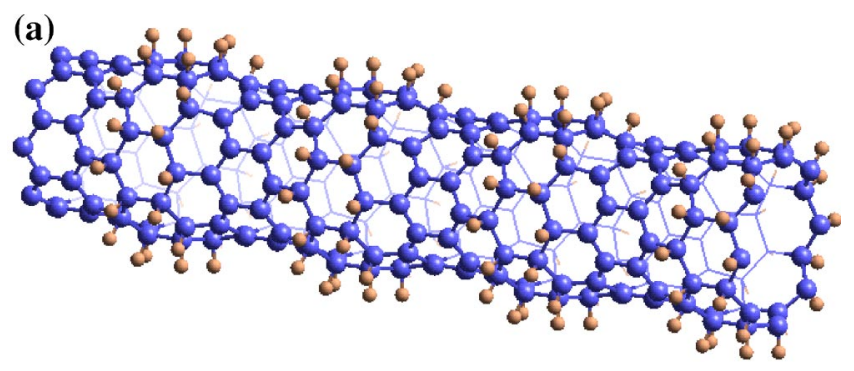

(b)

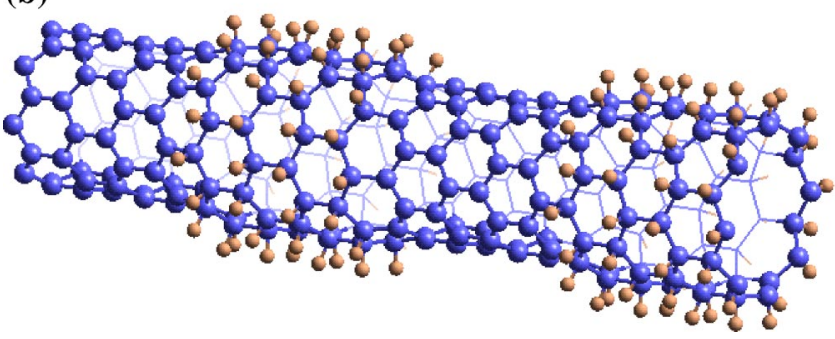

FIG. 1. Superlattice structures $\left[\left(\mathrm{C}_{32}\right)_{l}\left(\mathrm{C}_{32} \mathrm{H}_{32}\right)_{q}\right]$ formed on a single $(8,0)$ SWNT by modulating adsorption of hydrogen atoms. (a) $l=q=1$, (b) $l=q=2$. The positions of $64 \mathrm{H}$ and $128 \mathrm{C}$ atoms, as well as the superlattice parameter $c_{s}$, are calculated from full structure optimization by using conjugate gradient method. 
quantum structure formed from the SWNT). Note that owing to the relaxation of atomic structure upon hydrogenation, $c_{s}$ is not equal to integer multiples of $c, c$ being the optimized lattice constant of the bare $(8,0)$ SWNT.

In the present study we first treated two periodic quantum structures, i.e., $l=q=1$ and $l=q=2$ including, respectively, $64 \mathrm{C}$ and $32 \mathrm{H}$ atoms and $128 \mathrm{C}$ and $64 \mathrm{H}$ atoms in the supercell. The corresponding superlattice parameters were $c_{s} \sim 2 c$ and $c_{s} \sim 4 c$, respectively. Plane waves up to an energy cutoff of $500 \mathrm{eV}$ are used. With this energy cutoff and using ultrasoft pseudopotentials for carbon atoms, ${ }^{13}$ the total energy converges within $0.5 \mathrm{meV} / \mathrm{atom}$. Owing to the very large lattice constants of the supercell, k-point sampling is done only along the tube axis by using the Monkhorst-Pack special k-point scheme ${ }^{14}$ with $0.02 \AA^{-1}$ k-point spacing. Fully relaxed geometries are obtained by optimizing all atomic positions (as many as 96 for $l=q=1$ and 192 for $l$ $=q=2$ ) and the lattice constant (or superlattice parameter) $c_{s}$ along the tube axis until the maximum force and stress are less than $0.01 \mathrm{eV} / \AA$ and $0.1 \mathrm{GPa}$, respectively. Using the atomic structure determined from the optimization of the superlattice, $l=q=2$, in particular those of the atomic positions at the interface between hydrogenated and bare SWNT, we studied superlattices having larger lattice parameter. This way, we were able to treat relatively longer periodic quantum structures including large numbers of atoms (i.e., $120 \mathrm{C}$ and $64 \mathrm{H}$ atoms corresponding to $l=3, q=2$ and also $120 \mathrm{C}$ and $96 \mathrm{H}$ atoms corresponding to $l=2, q=3$, with $c_{s} \sim 5 c$ ) without further structure optimization. Hence, we have studied four different superlattices with different periodicities, i.e., $l+q=2,4$, and 5 .

\section{FORMATION OF QUANTUM STRUCTURES BY BAND-GAP MODULATION}

First we demonstrate how the electronic structure is modulated periodically in direct space so that a multiplequantum-well structure (MQWS) is generated on a prototype zigzag $(8,0)$ SWNT. The bare $(8,0)$ SWNT is a semiconductor with a direct band gap, $E_{g 0}=0.64 \mathrm{eV}$. Unsaturated $\pi^{*}$ states of carbons are active sites for the adsorption of hydrogen atoms. A single hydrogen atom is adsorbed on top of each carbon and completes the fourth covalent bond. The adsorption changes the planar $s p^{2}$ bonding on the cylindrical surface to a local $s p^{3}$-like bonding. The resulting structure is stable by an energy gain of $2.5 \mathrm{eV}$. This binding energy is relatively higher than the binding energy of $\mathrm{H}$ on the graphene due to curvature effects. ${ }^{12,15}$ Owing to the coupling between nearest-neighbor $\mathrm{H}$ atoms, saturation of all carbon atoms with hydrogens appears to be even more stable with an average binding energy $E_{b} \sim 2.7 \mathrm{eV} /$ atom. Exohydrogenation (i.e., saturation of all $\mathrm{C}$ atoms of a SWNT externally by $\mathrm{H}$ atom) gives rise to remarkable effects on the atomic and electronic structure. For example the $\mathrm{C}-\mathrm{C}$ bonds are lengthened, bond angles change, and the radius of the tube expands by $16 \%$. More importantly, the band gap of the bare $(8,0)$ tube increases from $E_{g 0}=0.64 \mathrm{eV}$ to $E_{g H}=2.1 \mathrm{eV}$. Moreover, it is found that the conductance is sensitive to hydrogen adsorption. ${ }^{16,17}$
In addition to the exohydrogenation, hydrogen decoration in different patterns and coverage can lead to a number of different physical properties. It has been shown that the electronic structure is strongly dependent on the pattern of external (exo) or alternating external and internal (exo-endo) adsorption of $\mathrm{H}$. Moreover, depending on the index $n$, the electronic structure of a $(n, 0)$ SWNT is modified differently under the same type-H decoration. ${ }^{9}$ For example, while the adsorption pattern, where the $\mathrm{C}$ atoms of the alternating zigzag rings of $\mathrm{C}-\mathrm{C}$ atoms are saturated by $\mathrm{H}$, makes the semiconducting $(8,0)$ SWNT a semimetal, the $(9,0)$ tube (which normally has a very small band gap) becomes a semiconductor with a large band gap.

Based on the above discussion, the zone of the SWNT which is kept clean and the zone which is hydrogenated are expected to have different band structure. This is the most crucial aspect that we have to explore. Normally, in the band continua, states in $\left(\mathrm{C}_{32}\right)_{l}$ (i.e., specified by $l$ ) zone are extended to the adjacent $\left(\mathrm{C}_{32} \mathrm{H}_{32}\right)_{q}$ (i.e., specified by $\left.q\right)$ zone. These are propagating (itinerant) states and have comparable integrated probability (or charge density), in both adjacent zones. Defining $\rho_{l}=\int_{l}\left|\Psi_{n, k}(\mathbf{r})\right|^{2} d \mathbf{r}$ and $\rho_{q}$ $=\int_{q}\left|\Psi_{n, k}(\mathbf{r})\right|^{2} d \mathbf{r}, \quad \rho_{l} \sim \rho_{q}$ for propagating states. On the other hand, if the energy of a state in one (say, in the hydrogen-free $l$ ) zone of the superlattice coincides with the band gap of the adjacent (hydrogenated $q$ ) zone, this particular state cannot propagate and has to be confined to the zone it belongs to. Accordingly, $\rho_{l} \gg \rho_{q}$ are very much different. In the past, these confined states have played a central role in optoelectronic device applications of MQWS's based on semiconductor superlattices such as GaAs-AlGaAs. ${ }^{1}$ At this point, the following three questions have to be addressed before we conclude whether MQWS's can be realized from the above superlattice structure: (i) Does the hydrogenated region persist or else adsorbed hydrogens can diffuse away to degrade the superlattice structure? (ii) What should be the extent of the zones $\left(\mathrm{C}_{32}\right)_{l}$ and $\left(\mathrm{C}_{32} \mathrm{H}_{32}\right)_{q}$ in order their electronic structure and hence their band gaps to be distinguishable from each other? (iii) How are the bands aligned, and hence how is the energy band diagram modulated along the nanotube?

Starting from the first question, we found that the fully hydrogenated zone $\left(\mathrm{C}_{32} \mathrm{H}_{32}\right)_{q}$ is stable, since $\mathrm{H}$ atoms remained bound to the carbon atoms at the end of full structure optimization. Our static calculation at $T=0 \mathrm{~K}$ did not yield diffusion of $\mathrm{H}$ towards the neighboring $\left(\mathrm{C}_{32}\right)_{l}$ regions. For the second question, the analysis of the charge density for various states suggests that indeed two different electronic structures can be realized in $l$ and $q$ zones of SWNT's, which are as short as two unit cells. The differences of the electronic structure become pronounced when the extension of $\left(\mathrm{C}_{32}\right)_{l}$ and $\left(\mathrm{C}_{32} \mathrm{H}_{32}\right)_{q}$ becomes longer. It is, therefore, concluded that an $(8,0)$ SWNT as short as a few nanometers is sufficient to make an electronic device. Our arguments are better clarified by the calculated total and local density of states in Fig. 2. Comparison of Figs. 2(a) and 2(b) shows how the band gap of a bare $(8,0)$ SWNT becomes wider as a result of exohydrogenation. The width of the valence band decreases by $\sim 2.5 \mathrm{eV}$, because the radius of the underlying 


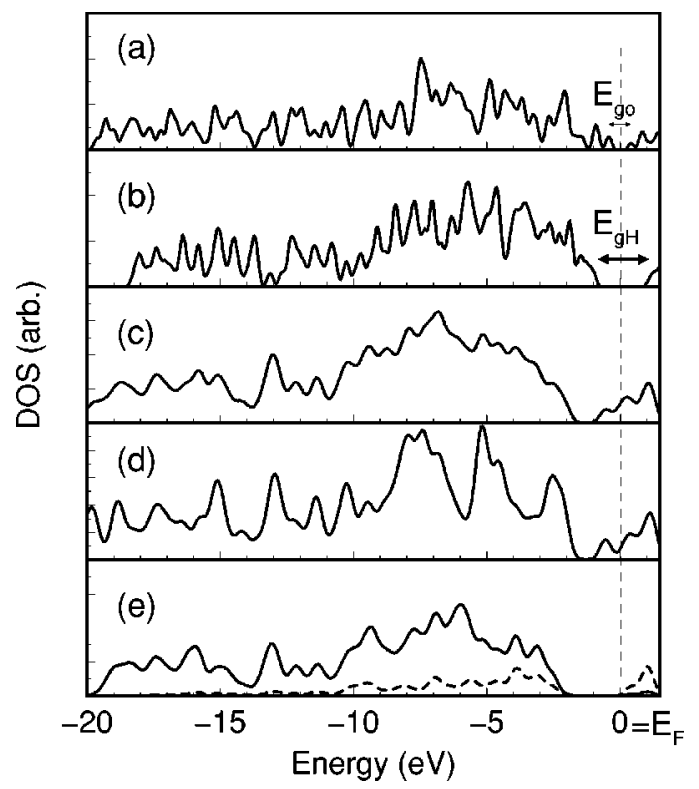

FIG. 2. (a) Total density of states of a bare $(8,0)$ SWNT, i.e., $l \rightarrow \infty$; (b) exohydrogenated $(8,0) \quad$ SWNT, i.e., $q \rightarrow \infty$; (c) $\left[\left(\mathrm{C}_{32}\right)_{2}\left(\mathrm{C}_{32} \mathrm{H}_{32}\right)_{2}\right]$ superlattice. (d) Local density of states of superlattice at $\left(\mathrm{C}_{32}\right)_{2}$; and (e) at $\left(\mathrm{C}_{32} \mathrm{H}_{32}\right)_{2}$ region with dashed curve indicating states derived from H. Band gaps $E_{g 0}$ (bare SWNT) and $E_{g H}$ (exohydrogenated SWNT) are also indicated.

hydrogenated tube increased by $16 \%$. Even if the total density of states of the superlattice $\left[\left(\mathrm{C}_{32}\right)_{2}\left(\mathrm{C}_{32} \mathrm{H}_{32}\right)_{2}\right]$ in Fig. 2(c) reflects the combination of state densities in Figs. 2(a) and 2(b), different zones display markedly different electronic structures. This can be revealed by calculating the local density of states, $\mathcal{L}(X, E)=\Sigma_{n, k} \int_{X} \Psi_{n, k}^{*}(\mathbf{r}) \Psi_{n k}(\mathbf{r}) \delta\left(E_{n, k}\right.$ $-E) d \mathbf{r}$. Here $X$ indicates either one of the zones ( $l$ or $q$ ). $\mathcal{L}\left[\left(\mathrm{C}_{32}\right)_{2}, E\right]$ presented in Fig. $2(\mathrm{~d})$ is similar to the state density of the bare $(8,0)$ in Fig. 2(a), except for some modifications in the gap region. On the other hand, we see from the local density of states of the hydrogenated region, $\mathcal{L}\left[\left(\mathrm{C}_{32} \mathrm{H}_{32}\right)_{2}, E\right]$ in Fig. 2(e), that the edge of the valence band is lowered and the band gap becomes slightly larger than $E_{g H}$. A significant contribution of states originating from hydrogen is found near the top of the valence band. We also note that $\mathcal{L}\left[\left(\mathrm{C}_{32} \mathrm{H}_{32}\right)_{2}, E\right]$ is well compared with the total density of states of a uniformly hydrogenated $(8,0)$ tube $(q \rightarrow \infty)$ in Fig. 2(b).

Further understanding of the electronic structure leading to the formation of MQWS can be obtained by examining energy bands and corresponding state charge densities. The population analysis indicates that electrons are transferred to $\left(\mathrm{C}_{32}\right)_{2}$ from $\left(\mathrm{C}_{32} \mathrm{H}_{32}\right)_{2}$. Propagating and confined states are easily distinguishable in Fig. 3. The charge density of a propagating state down in the valence band is shown in Fig. 3(a). The charge density of the highest valence band with energy $E=-0.52 \mathrm{eV}$ is shown in Fig. 3(b). This band can lead to the hole states confined to the quantum well in $\left(\mathrm{C}_{32}\right)_{2}$. The next band with energy $E=-0.03 \mathrm{eV}$ corresponds to a filled state which is confined to the quantum well in the conduction band of the $\left(\mathrm{C}_{32}\right)_{2}$ zone as shown in Fig. $3(\mathrm{c})$. It is responsible for the small structure appearing in Fig. (a)

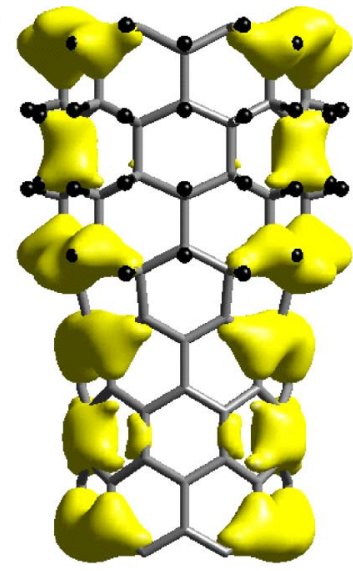

(c)

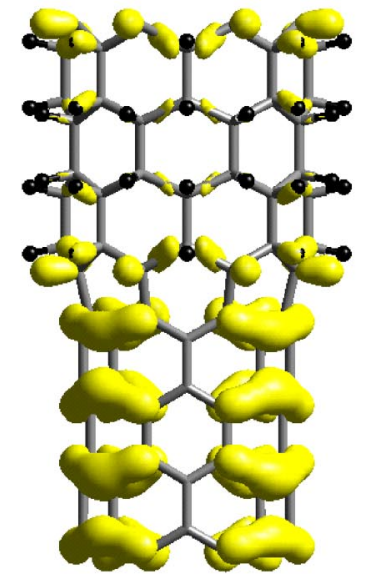

(b)

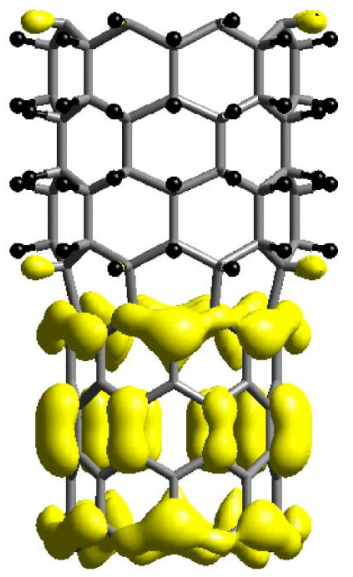

(d)

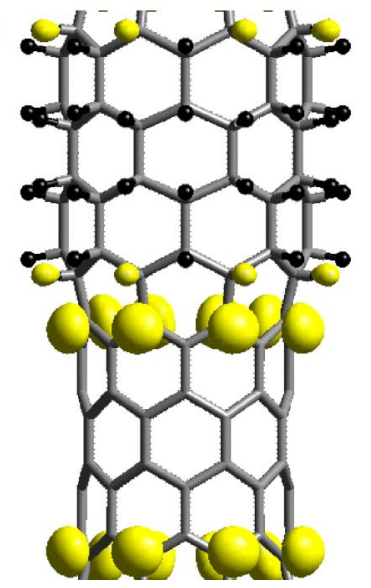

FIG. 3. Calculated self-consistent charge densities of propagating and confined states of the superlattice $\left[\left(\mathrm{C}_{32}\right)_{2}\left(\mathrm{C}_{32} \mathrm{H}_{32}\right)_{2}\right]$. See text.

2(d) just below the conduction-band edge of the $\left(\mathrm{C}_{32}\right)_{2}$ zone. As mentioned above, a similar feature occurs in the band gap of an infinite $\mathrm{C}_{32} \mathrm{H}_{18}$ SWNT, where alternating zigzag chains of $\mathrm{C}$ rings are saturated by $\mathrm{H}$ atoms. ${ }^{9}$ In the present case, the zigzag $\mathrm{C}-\mathrm{H}$ ring at the interface, adjacent to the $\left(\mathrm{C}_{32}\right)_{2}$ zone, is associated with this state. These states display almost no dispersion in the superlattice Brillouin zone. The charge density of a state down in the valence band, confined to the interface between two regions, is illustrated in Fig. 3(d). We found also states confined to either $\left(\mathrm{C}_{32}\right)_{2}$ or $\left(\mathrm{C}_{32} \mathrm{H}_{32}\right)_{2}$ in the valence and conduction bands. More confined states are formed, because the present superlattice is a one-dimensional system with small extension of $l$ and $q$, and hence offers a relatively small number of itinerant states propagating in both zones. By increasing the extension of two different regions the confinement of the states and the energy band diagram in direct space become pronounced. Nevertheless, the above analysis clearly indicates that a MQWS with markedly different band gaps is generated.

The formation of different zones leading to different electronic structures on the same $(8,0)$ SWNT upon modulating $\mathrm{H}$ adsorption and also the effect of superlattice size (i.e., $l, q$, and $l+q$ ) are further analyzed by examining the electronic potential. The electronic potential is calculated selfconsistently and consists of Hartree, exchange, and ionic 


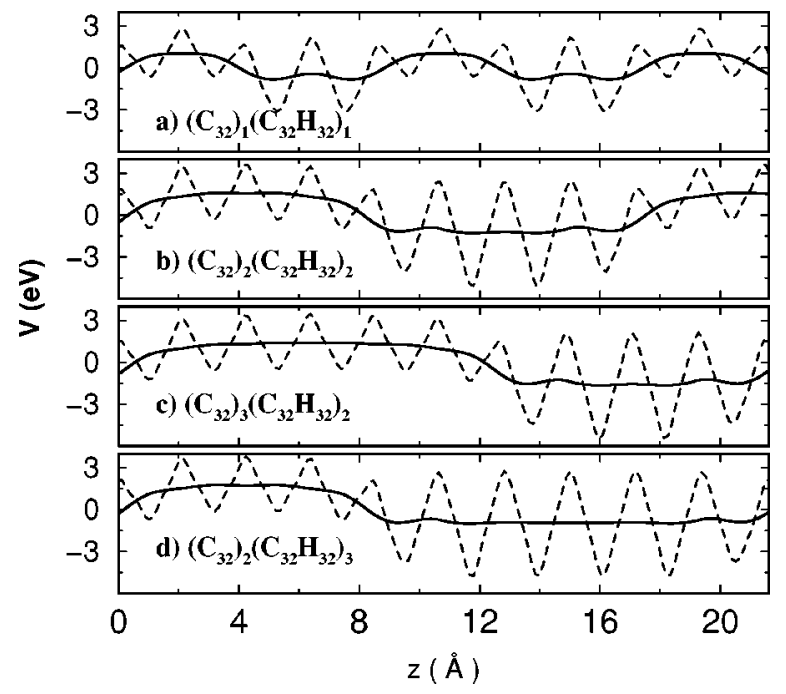

FIG. 4. Averages of calculated self-consistent electronic poten-

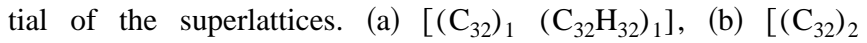

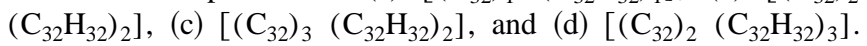
Dashed and thick lines are planarly averaged $\bar{V}_{e}(z)$ and running averaged $\bar{V}_{r, e}(z)$, respectively. Relatively higher-cell-averaged potential zones correspond to $\left(\mathrm{C}_{32}\right)_{l}$. See text.

pseudopotentials, $V_{e}(\mathbf{r})=V_{H}(\mathbf{r})+V_{x}(\mathbf{r})+V_{\text {ion }}(\mathbf{r})$. In Fig. 4 we present the planarly averaged electronic potential

$$
\bar{V}_{e}(z)=\frac{1}{a_{s} b_{s}} \int V_{e}(x, y, z) d x d y
$$

and running average of the planarly averaged potential $\bar{V}_{r, e}(z)$. Here $z$ is parallel to the axis of SWNT, and in planarly averaging the integral is evaluated on the $x y$ plane in the supercell. The running average is the average of $\bar{V}_{e}(z)$ between $z=z_{i}$ and $z=z_{i}+z_{0}, z_{0}$ being an appropriate subperiodicity in the cell, such as the peak-to-peak distance of $\bar{V}_{e}(z)$. As seen in Fig. 4(a), the short periodicity $l=q=1$ is not sufficient to induce two adjacent zones with markedly different electronic structures. The zone corresponding to exohydrogenated SWNT's is mixed with the interface region shown by a small peak, and the potential difference between two zones, $\Delta V$, is not too large. In Fig. 4(b) the differences between two zones become pronounced, and $\Delta V$ increases for the superlattice, $l=q=2$. Figures 4(c) and 4(d) indicate that the potential $\bar{V}_{r, e}(z)$ becomes flatter as $l$ and $q$ increase from 2 to 3 , and two adjacent zones display markedly different electronic structure. Increasing flattening of $\bar{V}_{r, e}(z)$ with increasing $l$ and $q$ justifies our argument that the formation of quantum structures becomes pronounced for large superlattice periodicity. We calculated $\bar{V}_{r, e}(1.0 \mathrm{eV},-0.7 \mathrm{eV}),(1.56$ $\mathrm{eV},-1.08 \mathrm{eV}),(1.37 \mathrm{eV},-1.61 \mathrm{eV})$, and $(1.75 \mathrm{eV}$, $-0.94 \mathrm{eV})$ for the superlattices $(l=1, q=1),(l=2, q$ $=2),(l=3, q=2)$, and $(l=2, q=3)$, respectively. Consequently, for the same superlattices $\Delta V$ is found to be $1.7 \mathrm{eV}$, $2.64 \mathrm{eV}, 2.98 \mathrm{eV}$, and $2.69 \mathrm{eV}$. The charge transfer from the hydrogenated to hydrogen-free zone is the primary cause of why $\bar{V}_{r, e}$ has raised at the hydrogen-free zone to yield a large $\Delta V$.

\section{CALCULATION OF BAND OFFSETS}

Now the determination of the band offsets leading to the energy band diagram with quantum wells and barriers is our next task. Band lineup of zones $\left(\mathrm{C}_{32}\right)_{l}$ and $\left(\mathrm{C}_{32} \mathrm{H}_{32}\right)_{q}$ is a complex process involving the relaxation of the atomic structure and charge transfer at the interface and, also, resulting in the modification of the electronic potential. Experimental and theoretical methods have been proposed in the past to determine the band offsets and, hence, to obtain a band diagram perpetuating along the semiconductor heterostructures. A solution of this problem ${ }^{18}$ involves the following steps: First, the average electronic potentials corresponding to $\bar{V}_{r, e}(z)$ for finite $l$ and $q$, as well as for infinite $\left(\mathrm{C}_{32}\right)_{l=\infty}$ and $\left(\mathrm{C}_{32} \mathrm{H}_{32}\right)_{q=\infty}$, are calculated. The actual atomic positions, especially those at the interface, and the charge transfer between regions are of crucial importance for the band offsets and are incorporated self-consistently in the calculated average electronic potential. Second, for the case of $l=q=\infty$ the energy difference between the edge of the valence band $E_{V}$ and average potential-i.e., $\left.\Delta E_{B}\right|_{l(q)=\infty}=E_{V, l(q)=\infty}$ $-\left.\bar{V}_{r, e}\right|_{l(q)=\infty}$-are calculated. We found $\Delta E_{B}$ for $\left(\mathrm{C}_{32}\right)_{l=\infty}$ and $\left(\mathrm{C}_{32} \mathrm{H}_{32}\right)_{q=\infty}$ to be $2.62 \mathrm{eV}$ and $3.91 \mathrm{eV}$, respectively. In the third step, one assumes that $\Delta E_{B}$ is unchanged for finite $l$ and $q$, and determines the valence-band edges of a particular superlattice with given $l$ and $q$ by adding $\left.\Delta E_{B}\right|_{l(q)=\infty}$ to the corresponding average potential values $\bar{V}_{r, e}$ of the $l$ and $q$ zones. At the end, the energies of the valence-band edges of two adjacent zones (i.e., hydrogen free and exohydrogenated) are fixed. Then, the valence-band offset of the superlattice is $\Delta E_{V}=E_{V, l}-E_{V, q}$. The energies of the conductionband edges of the superlattice are determined by adding band gaps of different zones (calculated for $l=\infty$ and $q=\infty$ ) to the energies of the valence-band edges, i.e., $E_{C, l}=E_{V, l}+E_{g 1}$ and $E_{C, q}=E_{V, q}+E_{g 2}$. Here, $E_{g 1}=E_{g 0}$ and $E_{g 2}=E_{g H}$ in Fig. 2. The conduction-band offset is then determined by subtracting these latter energies, i.e., $\Delta E_{C}=E_{C, q}-E_{C, l}$.

We note that the above procedure used to determine the band diagram of heterostructures may involve error bars in the short-periodicity superlattices, such as $l, q=1$. In these superlattices $\bar{V}_{r, e}(z)$ is not flat, but exhibits a small variation with $z$. As seen in Fig. 2, the band gaps in the zones of small $l$ and $q$ can differ from the band gaps calculated for $l=q$ $=\infty$. Moreover, $\left.\Delta E_{B}\right|_{l(q)}$ may be different from the corresponding values $\left.\Delta E_{B}\right|_{l(q)}$. At least, $\Delta E_{B}$ varies with $z$ for small $l$ and $q$. Since the self-consistent-field (SCF) calculation of large-periodicity superlattices cannot be achieved with the present computational tools and our objective is only to show whether quantum structures can form, we apply the above procedure and use the results of our superlattice calculations to estimate the band offsets. In Fig. 5 we present our estimated values of band offsets for the superlattices ( $l$ $=q=2),(l=3 ; q=2)$, and $(l=2 ; q=3)$. The superlattice $(l=q=1)$ does not allow us to make any estimation because 


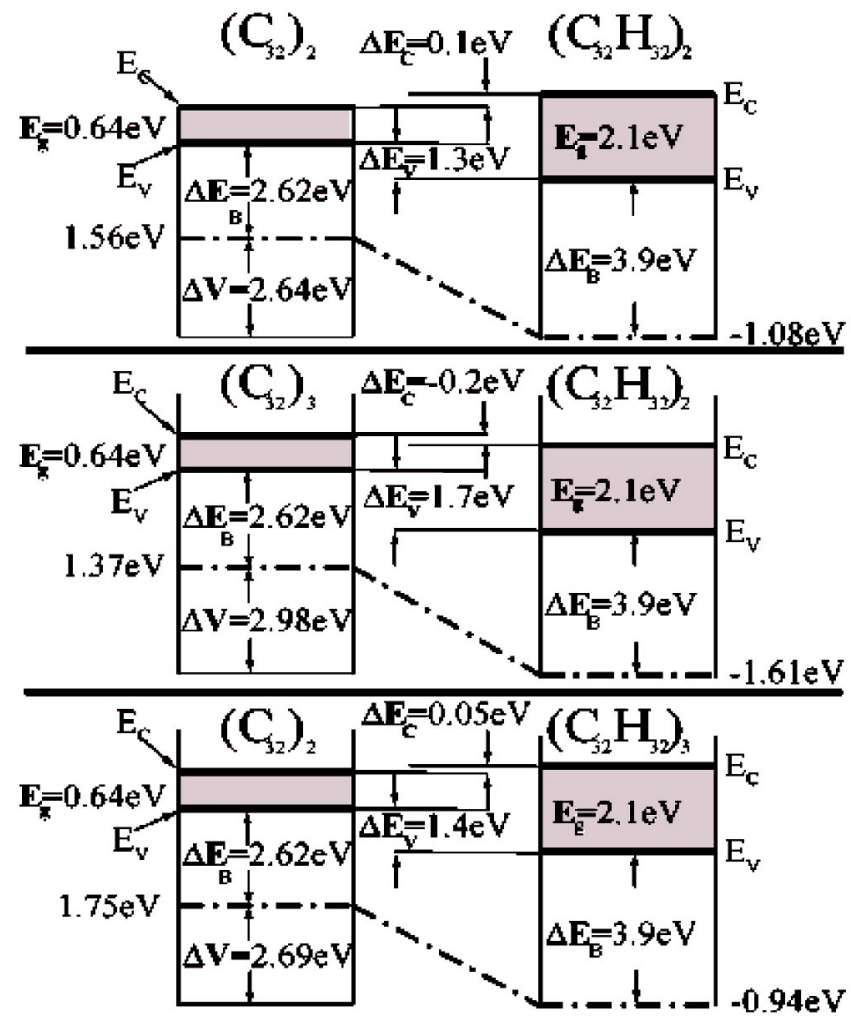

FIG. 5. Schematic illustration of the energy band diagram and band offsets estimated by using averaged self-consistent electronic potentials $\bar{V}_{r, e}(z)$ calculated for the $l$ and $q$ zones, $\Delta E_{B, l(q)}$, and band gaps $E_{g 1}$ and $E_{g 2}$ calculated for $l=\infty$ and $q=\infty$.

of the uncertainty in the determination of $\bar{V}_{r, e}(z)$. For $(l$ $=q=2$ ), $\Delta E_{C}$ and $\Delta E_{V}>0$ indicate a type-I (normal) band lineup and give rise to quantum wells in the conduction as well as in the valence band of the $\left(\mathrm{C}_{32}\right)_{2}$ zone. This band diagram is consistent with the confinement of states in Figs. $3(\mathrm{~b})$ and 3(c). It appears that the band gap $E_{g, l=2}<E_{g, l=\infty}$, and the first band with $E=-0.03 \mathrm{eV}$ is confined in the quantum well formed in the conduction band of $\left(\mathrm{C}_{32}\right)_{2}$ as shown in Fig. 3(c). The band lineup of $(l=3, q=2)$ is type II (staggered), while $(l=2, q=3)$ displays a normal band lineup. We note that alternation of band lineups with superlattice periodicity originates from small values of $\left|\Delta E_{C}\right|$. For long superlattice periodicity with large $l$ and $q$ it is expected that $\Delta E_{V} \sim 1.4 \mathrm{eV}$ and $E_{C, l} \sim E_{C, q}$.

We can check our estimated values by taking a more direct and practical approach and deduce the band offsets and resulting energy band diagram from the calculated electronic structure of the superlattice $l=q=2$. The energy band diagram in direct space is obtained by analyzing the characters and energy positions of the band structure and by examining the gaps of the local density of states shown in Fig. 2. Shifts of the bands corresponding to $\left(\mathrm{C}_{32}\right)_{2}$ and $\left(\mathrm{C}_{32} \mathrm{H}_{32}\right)_{2}$ zones and the resulting band offsets are already incorporated in the local density of states in Figs. 2(d) and 2(e). The band gaps at the hydrogen-free and exohydrogenated regions of the superlattice are different from the values corresponding to $l$ $=\infty$ and $q=\infty$ and are deduced from the local density of states to be $E_{g, l=2} \sim 0.5 \mathrm{eV}$ and $E_{g, q=2} \sim 2.3 \mathrm{eV}$, respec-

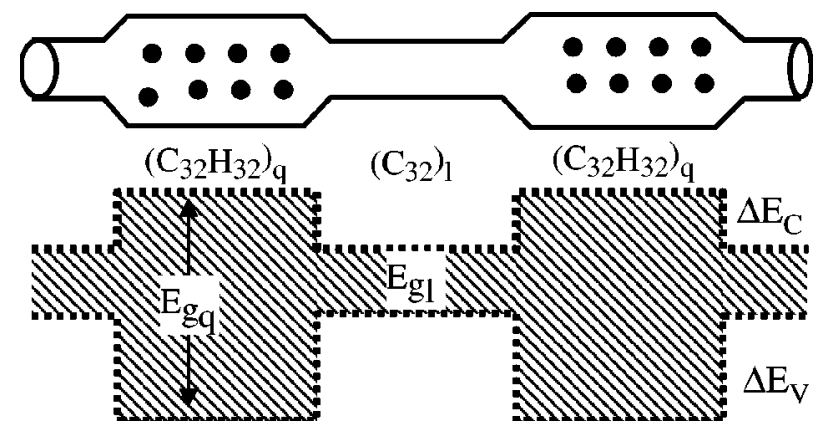

FIG. 6. Schematic illustration of the energy band diagram of a resonant tunneling double-barrier structure generated by a modulating adsorption of $\mathrm{H}$ atoms on an s-SWNT superlattice indicating a type-I, normal band lineup.

tively. The band offsets at the valence and conduction band are estimated to be $\Delta E_{V}=\sim 1.4 \mathrm{eV}$ and $\Delta E_{C}=\sim 0.4 \mathrm{eV}$, respectively. Keeping in mind that the value of $\Delta E_{C}$ is small and it may be within the error bars in short-periodicity superlattices, both procedures are seen to yield similar type of band alignment, i.e., type-I, normal band lineup.

\section{DISCUSSION AND CONCLUSIONS}

The present discussion for the periodically repeating quantum wells can be extended to finite systems-for example, to the resonant tunneling double barriers - made by a zone $\left(\mathrm{C}_{32}\right)_{l}$ placed between two zones of $\left(\mathrm{C}_{32} \mathrm{H}_{32}\right)_{q}$, i.e., $\left(\mathrm{C}_{32} \mathrm{H}_{32}\right)_{q}\left(\mathrm{C}_{32}\right)_{l}\left(\mathrm{C}_{32} \mathrm{H}_{32}\right)_{q}$. A schematic description of the band-gap diagram is presented in Fig. 6. Both $\Delta E_{V}>0$ and $\Delta E_{C}>0$ give rise to a type-I, normal band lineup as described in the figure.

Metallic reservoirs adjacent to both barriers, i.e., $\left(\mathrm{C}_{32} \mathrm{H}_{32}\right)_{q}$, and metallic connects of the device can be achieved by metal coating $^{19,20}$ or by oxygenation ${ }^{21,22}$ of the same tube. Also a metal-semiconductor heterostructure can be formed by modulating the hydrogenation of a $(n, n)$ armchair SWNT. This way metallic connects are provided by the hydrogen-free ends of the tube.

The hydrogenation of SWNT's appears to be a difficult process, since the $\mathrm{H}_{2}$ molecule does not form strong chemical bonds at the surface of the tube, but is bound mainly by a weak van der Waals interaction. Thus, adsorption of atomic hydrogen upon the breaking of $\mathrm{H}_{2}$ on the SWNT surface cannot occur. Under these circumstances, exohydrogenation can be achieved by exposing the SWNT to atomic hydrogen. Such experiments with atomic hydrogen have been performed previously. Our recent calculations provide also evidence that atomic $\mathrm{H}$ adsorption can follow the breaking of molecules when the high-curvature sites of radially deformed SWNT's are bombarded with $\mathrm{H}_{2}$ molecules. Nevertheless, we believe that modulating $\mathrm{H}$ atom adsorption can be achieved by masking the zones which are desired to be free of hydrogen atom. We also note that quantum structure formation may not be restricted to the adsorption of $\mathrm{H}$ atoms. It has been shown that adsorption of transition-metal atoms on SWNT's gives rise to a magnetic ground state. ${ }^{20}$ It is ex- 
pected that one can produce magnetic quantum structures by the decoration of the tube through the adsorption of transition-metal atoms, such as Ti, Mo, etc.

In conclusion, our results obtained from first-principles calculations demonstrate that one can generate a variety of quantum structures by changing the sequence and $l$ and $q$ extension, and also by applying different patterns of hydrogenation on different kinds of SWNT's.

\section{ACKNOWLEDGMENTS}

This work was partially supported by the NSF under Grant No. INT01-15021 and TUBITAK under Grant No. TBAG-U/13(101T010). S.C. acknowledges partial support from the Academy of Science of Turkey (TÜBA). S.C. thanks Sefa Dag for his assistance in the preparation of the paper.

${ }^{1}$ Highlights in Condensed Matter Physics and Future Prospects,

(1992). edited by L. Esaki, Vol. 285 of NATO Advanced Study Institute (Plenum Press, New York, 1991).

${ }^{2}$ S. Iijima, Nature (London) 354, 56 (1991).

${ }^{3}$ P.G. Collins, A. Zettl, H. Bando, A. Thess, and R.E. Smalley, Science 278, 100 (1997).

${ }^{4}$ J. Kong, N.R. Franklin, C. Zhou, M.G. Chapline, S. Peng, K. Cho, and H. Dai, Science 287, 622 (2000).

${ }^{5}$ L. Chico, V.H. Crespi, L.X. Benedict, S.G. Louie, and M.L. Cohen, Phys. Rev. Lett. 76, 971 (1996).

${ }^{6}$ A. Bezryadin, A.R.M. Verschueren, S.J. Tans, and C. Dekker, Phys. Rev. Lett. 80, 4036 (1998).

${ }^{7}$ V. Derycke, R. Martel, J. Appenzeller, and Ph. Avouris, Phys. Rev. Lett. 80, 2773 (2002).

${ }^{8}$ T. Yildirim, O. Gülseren, and S. Ciraci, Phys. Rev. B 64, 075404 (2001).

${ }^{9}$ O. Gülseren, T. Yildirim, and S. Ciraci, Phys. Rev. B 66, 121401 (2002).

${ }^{10}$ M.C. Payne, M.P. Teter, D.C. Allen, T.A. Arias, and J.D. Joannopoulos, Rev. Mod. Phys. 64, 1045 (1992).

${ }^{11}$ J.P. Perdew, J.A. Chevary, S.H. Vosko, K.A. Jackson, M.R. Pederson, D.J. Singh, and C. Fiolhais, Phys. Rev. B 46, 6671

${ }^{12}$ O. Gülseren, T. Yildirim, S. Ciraci, and C. Kilic, Phys. Rev. B 65, 155410 (2002); O. Gülseren, T. Yildirim, and S. Ciraci, ibid. 65, 153405 (2002).

${ }^{13}$ D. Vanderbilt, Phys. Rev. B 41, 7892 (1990).

${ }^{14}$ H.J. Monkhorst and J.D. Pack, Phys. Rev. B 13, 5188 (1976).

${ }^{15}$ O. Gülseren, T. Yildirim, and S. Ciraci, Phys. Rev. Lett. 87, 116802 (2001).

${ }^{16}$ A.N. Andriotis, M. Menon, D. Srivastava, and G.E. Froudakis, Phys. Rev. B 64, 193401 (2001).

${ }^{17}$ A.N. Andriotis, M. Menon, and D. Srivastava, J. Chem. Phys. 117, 2836 (2002).

${ }^{18}$ L. Colombo, R. Resta, and S. Baroni, Phys. Rev. B 44, 5572 (1991).

${ }^{19}$ V.M.K. Bagci, O. Gülseren, T. Yildirim, Z. Gedik, and S. Ciraci, Phys. Rev. B 66, 045409 (2002).

${ }^{20}$ E. Durgun, S. Dag, V.M.K. Bagci, O. Gülseren, T. Yildirim, and S. Ciraci, Phys. Rev. B 67, 201401 (2003).

${ }^{21}$ P.G. Collins, K. Bradley, M. Ishigami, and A. Zettl, Science 287, 1801 (2000).

${ }^{22}$ S. Dag, O. Gülseren, T. Yildirim, and S. Ciraci, Phys. Rev. B 67, 165424 (2003). 